\title{
Lattice structures under uniaxial impact loads: An experimental study
}

\author{
Alexandre Riot ${ }^{1,2^{*}}$, Enrico Panettieri ${ }^{1}$, Marco Montemurro ${ }^{1}$, Sandra Guerard ${ }^{1}$, Jérémie \\ Girardot $^{1}$ and Antonio Cosculluela ${ }^{2}$ \\ ${ }^{1}$ Arts et Métiers Institute of Technology, Université de Bordeaux, CNRS, INRA, Bordeaux INP, \\ HESAM Université, I2M UMR 5295, F-33405 Talence, France \\ ${ }^{2}$ CEA-DAM/CESTA, 15 Avenue des Sablières, 33114 Le Barp, France
}

\begin{abstract}
Innovative additive manufacturing processes open new design opportunities, in the field of periodic cellular shock absorbers. In this work, an experimental campaign on lattice samples has been developed and carried out for intermediate velocities. The geometries of the lattice samples used in the experimental tests are the result of a sensitivity analysis conducted in a previous work. As a complement to the results provided by the numerical model used in the sensitivity analysis, the experimental tests highlighted an aspect of capital importance: the geometry maximizing energy absorption is highly dependent on the applied strain. Furthermore, local buckling effects, induced primarily at the sample's surfaces in contact with the support of the testing machine, induce a stiffness decrease for specific geometries, thus reducing the absorbed energy. This study emphasises the importance of geometry selection, to tailor specific mechanical responses.
\end{abstract}

\section{Introduction}

The recent improvements made in the additive manufacturing (AM) processes led design methodologies towards innovative solutions, either generated from the ground up through topology optimization ([1],[2]), or directly improved through its parameters using parametric optimization ([3],[4]). Therefore, design approaches lean progressively towards technological solutions that could not be manufactured through conventional manufacturing processes, such as periodic cellular structures. The design of such cellular materials conventionally named lattices materials (LMs) - may be used to tailor specific mechanical properties adapted to different types of load cases, such as impacts or shock absorption. Stochastic cellular structures are described by arbitrary mesoscopic geometry, such as foam or wood-based materials. Currently, these materials, with high energy absorption capacity (EAC) are commonly used as shock absorbers [5]. This study presents the results of an experimental campaign carried out on specimens made of multiple lattice cells and tested under compressive dynamic load at different strain rate value. These results allow for a first experimental assessment to be obtained on the influence of the LM representative volume

*Corresponding author: alexandre.riot@ensam.eu 
element (RVE) topology on the energy absorption capability and it allows to establish an experimental reference to be used for the validation of numerical models.

\section{Materials and methods}

\subsection{Geometry and material properties}

Energy absorption for shock absorbers may be quantified using the EAC as a primary indicator:

$$
W_{\mathrm{EAC}}=\frac{1}{\rho_{\text {bulk }}} W_{0}=\frac{1}{\rho_{\text {bulk }}} \int_{0}^{\varepsilon_{0}} \sigma(\varepsilon) d \varepsilon,
$$

where $W_{0}$ is the absorbed energy per volume unit, $\rho_{\text {bulk }}$ is the bulk material's density, $\varepsilon_{0}$ is the applied strain to the sample and $\sigma$ is the uni-axial stress. A preliminary study on the EAC of different LM RVE architectures constituted of an elastomeric bulk material has been carried out in order to determine the RVE topology showing the highest EAC. A total of four lattice geometries have been chosen, and investigated, as shown in Fig. 1, among the LM architectures available in a given database [6]. The sensitivity analysis has been carried out using the nonlinear dynamics implicit solver in ABAQUS, through python batch commands. Conclusions of the sensitivity analysis indicate that the EAC is significantly affected by the LM RVE topology. From these results, the truncated cuboctahedron 2+ (TrCO) cell seems to have the highest EAC among the considered cells in the assumed range of strain-rate. For further insight in the numerical analyses, the reader is addressed to [6].

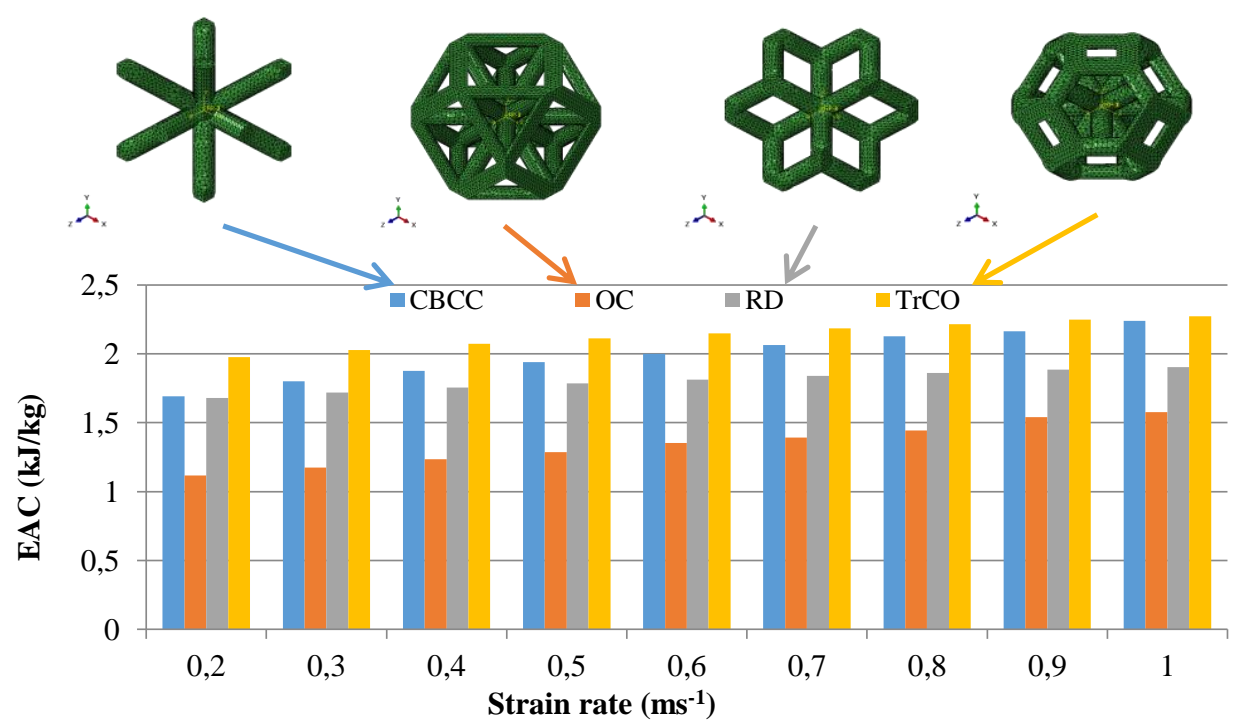

Fig. 1. Preliminary EAC assessment of different geometries for a strain $\varepsilon_{0}=0,5$

In the present study, the lattice specimens are made of $5 \times 5 \times 5$ cubic lattice cells, with a 6 $\mathrm{mm}$ cell width and with a relative density of $20 \%$. For the experiments, the lattice samples are manufactured using photopolymerization, more specifically Digital Light Synthesis, on a M2 Carbon3D additive manufacturing printer. The bulk material used for the sample manufacturing, referred as the MIX90A, is a resin resulting of the combination of two 
types: a rigid polyurethane resin (RPU70) and an elastomeric polyurethane resin (EPU40). The constitutive law of the MIX90A material is modelled with an isotropic-hardening elastic-plastic law with strain rate effects and ductile damage, using a Cowper-Symonds (CS) constitutive law:

$$
\sigma_{\mathrm{D}}=\sigma_{\mathrm{QS}}\left(1+\frac{\dot{\varepsilon}}{D}\right)^{\frac{1}{p}}
$$

where $\sigma_{\mathrm{D}}$ is the dynamic stress, $\sigma_{\mathrm{QS}}$ is the quasi-static stress, $\dot{\varepsilon}$ is the strain rate, and $D$ and $p$ the CS law parameters. These parameters have been determined using experimental data from quasi-static and dynamic mechanical tests carried out on samples fully made of bulk material. The MIX90A material properties are presented in Table 1 and Table 2.

Table 1. MIX90A material properties

\begin{tabular}{lr}
\hline \hline Properties & Values \\
\hline \hline Young Modulus $(\mathrm{GPa})$ & $36 \times 10^{-3}$ \\
Poisson ratio & 0.45 \\
Bulk density $\left(\mathrm{kg} / \mathrm{mm}^{3}\right)$ & $1.053 \times 10^{-6}$ \\
Fracture strain for ductile damage & 2.9 \\
Stress triaxiality & 0.33 \\
Strain rate fracture $\left(\mathrm{ms}^{-1}\right)$ & $1 \times 10^{-6}$ \\
\hline \hline$C S$ parameters & \\
\hline$D\left(\mathrm{~ms}^{-1}\right)$ & $100 \times 10^{-3}$ \\
$p$ & 0.69 \\
\hline
\end{tabular}

Table 2. MIX90A

plastic properties

\begin{tabular}{lr}
\hline $\begin{array}{l}\text { Plastic } \\
\text { strain }\end{array}$ & $\begin{array}{r}\text { Yield stress } \\
(\mathrm{MPa})\end{array}$ \\
\hline \hline 0 & 1.005 \\
0.1 & 2.089 \\
0.3 & 2.784 \\
0.6 & 3.357 \\
0.9 & 3.859 \\
1.3 & 4.560 \\
1.7 & 5.444 \\
1.9 & 5.919 \\
2.5 & 7.631 \\
\hline
\end{tabular}

\subsection{Experimental methodology}

The experiment represents an impact-like scenario at constant velocity. The experimental bench consists of an electromagnetic actuator, mounted on a massive table (c.f. Fig. 2). To carry out the dynamic compression test at constant velocity, the velocity time history of the actuator is defined as shown in Fig. 3. In particular, a compression phase and relaxation phase can be identified. The initial position of the actuator is chosen in order to obtain an initial actuator velocity, $V_{\text {act }}$ of $0,5 \mathrm{~m} / \mathrm{s}$. Compression force is measured using a piezoelectric sensor, mounted on the compression plateau. The experiment is captured using a high speed camera, and displacement is determined using DIC on the recovered images. From this data, global strain and equivalent stress (divided by the sample's crosssection) are computed and used for comparison purposes with the results provided by the numerical model [6].

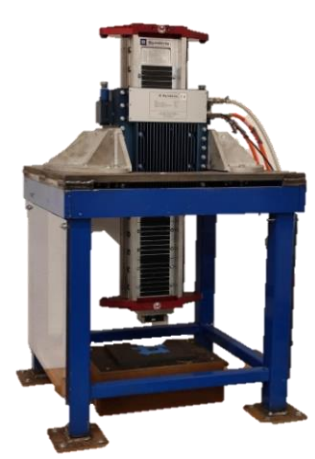

Fig. 2. Electromagnetic actuator

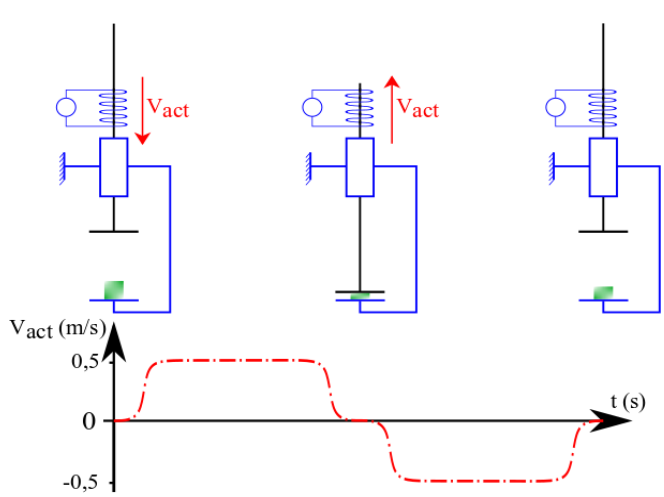

Fig. 3. Simplified experimental layout and associated speed diagram 


\section{Experimental Results}

Experiments are carried out for each RVE topology on three different samples in order to assess the experiment's repeatability. Results for each compression tests are illustrated in Fig. 4: each graph represents the average strain/equivalent stress curves for each sample, and the standard deviation associated to each point of measurement. Equivalent stress has been computed dividing the force by the global sample's cross-section, $S_{\text {samp }}$ equal to $900 \mathrm{~mm}^{2}$. EAC results for each lattice topology are reported in Table 3, and absorbed energy per volume unit are plotted compared to the peak stress $\sigma_{p}$ in Fig. 5. Both axes are normalised by the MIX90A bulk modulus $E_{\mathrm{MIX} 90 \mathrm{~A}}$, as suggested in [5].
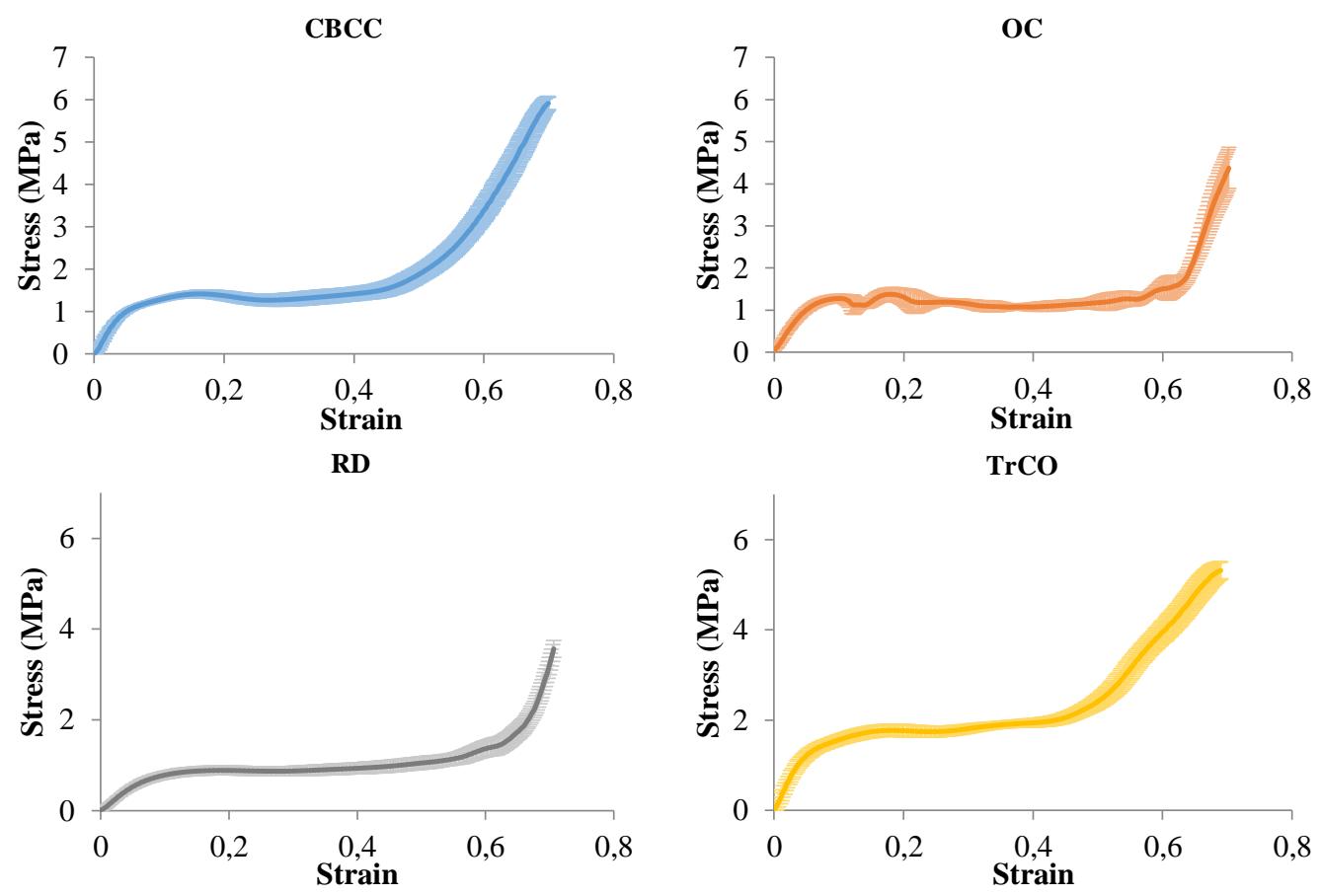

Fig. 4. Experimental results: averaged mechanical responses and their standard deviation

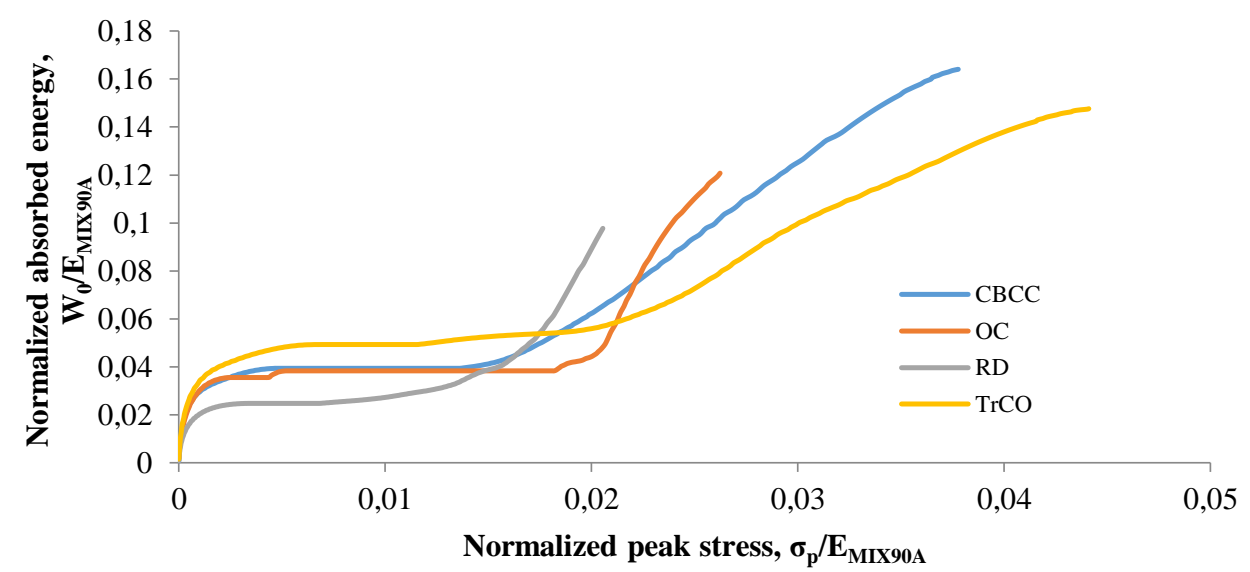

Fig. 5. Energy absorption diagram at relative density $20 \%$ for a strain $\varepsilon_{0}=0.7$ 
Table 3. EAC values for each cellular structure

\begin{tabular}{|c|c|c|c|c|}
\hline & $\mathrm{CBCC}$ & $\mathrm{OC}$ & $\mathrm{RD}$ & $\mathrm{TrCO}$ \\
\hline 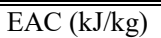 & (0.24 & 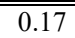 & 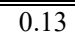 & $\bar{~} 0.28$ \\
\hline
\end{tabular}

According to [5], the curves' tendencies displayed in Fig. 4 and Fig. 5 appear to be representative of elastomeric foams, typically described by an elastic phase, followed by a plateau and densification. In particular in Fig. 5, since each RVE topology is unique, each curve presents different envelopes and shoulder heights, the highest being the most efficient. For instance, the OC samples responses tend to have an oscillatory tendency around the plateau. According to [7], local buckling may be the cause of such local variation. Indeed, when looking closer into the OC lattice structure in Fig. 6, it is clear that the first layer of RVEs in contact with the actuator's pressure plate buckles first, which confirms the influence of dynamic buckling on the structure dynamical response. The second layer of RVEs undergoing local buckling is the last layer of the sample. However, the local buckling occuring on the last layer of RVEs seems to have a lower impact on the structure dynamical behaviour. Furthermore, such behaviours are occur in all three OC samples, which indicates that buckling is likely to happen first on the sample's surface directly hit by the pressure plate and then on the specimen opposite side.
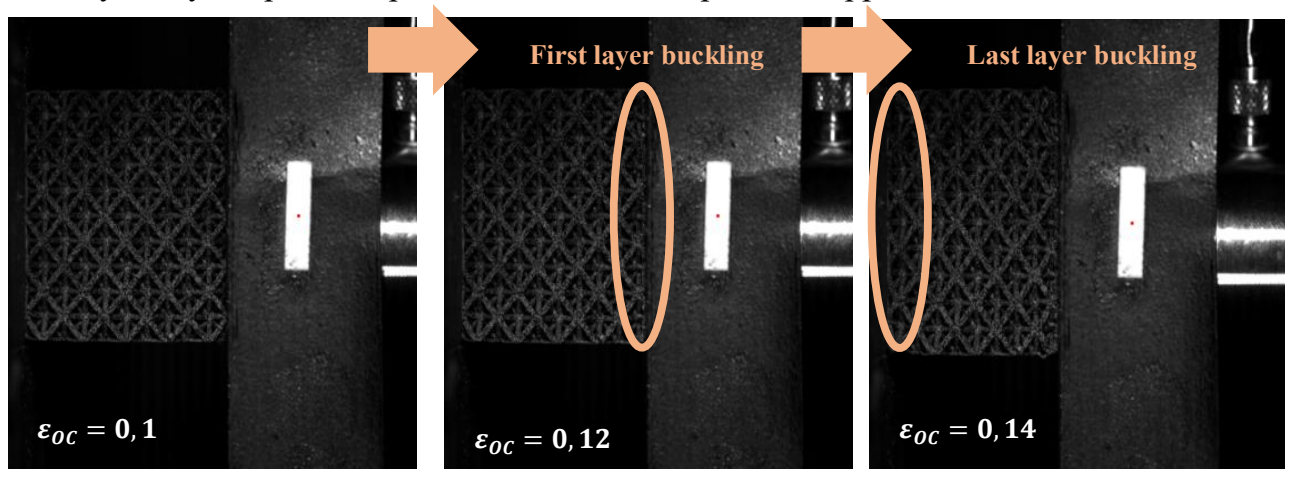

Fig. 6. OC cell : local buckling occurs in both first and last layers of RVEs

In contrast, the RD sample presents a very smooth transition among the different phases, and although it is subject to local buckling at the actuator compression plateau. Indeed, when analysing the recorded images shown in Fig. 7, the first cell layer buckles, but this does not seem to significantly affect the macroscopic EAC because in the following phases the subsequent layers of RVEs undergo a homogeneous compression.
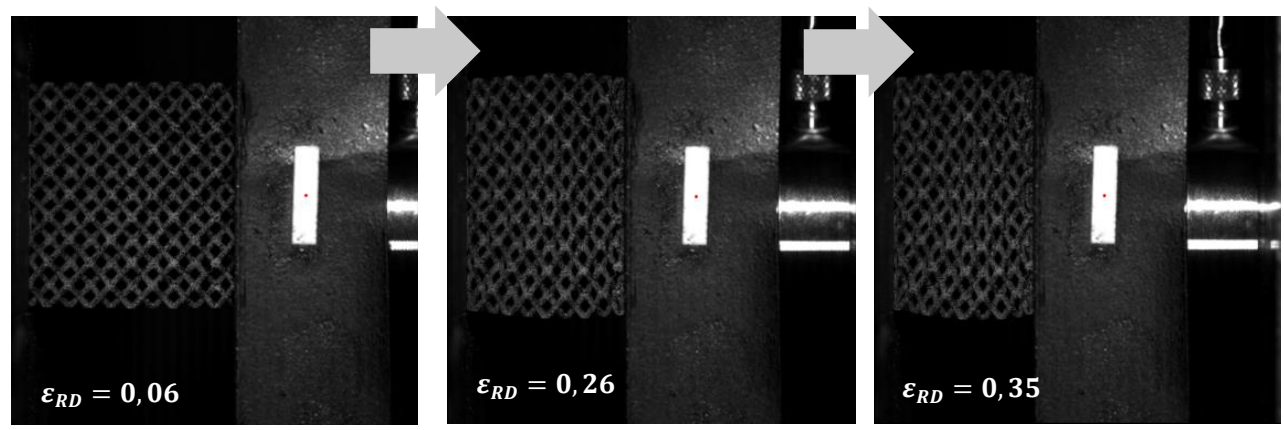

Fig. 7. RD cell : the first layer of RVEs experiences local buckling followed by a homogeneous compression state in each layer 


\section{Conclusions and prospects}

The experimental results presented in this work highlighted the importance of choosing the right LM RVE topology, for a specific strain and strain-rate, applied to medium velocity impact tests. According to the results of the experiements, the $\mathrm{TrCO}$ lattice geometry seems to exhibit the highest energy absorption capability as suggested by the numerical sensitivity analysis. However, it is important to emphasize that the CBCC cell reaches densification faster, which indicates that the CBCC cell is capable of higher energy absorption capacity at higher values of strain when compared to other geometries. The experimental results suggest that the LM RVE topology can be used as further design parameter allowing for different mechanical responses to be included within a structure at different regions to efficiently cope with given structural requirements. As far as prospects of this work are concerned, further experimental tests will be carried out to better understand the role of the local buckling and its influence on the EAC for each LM RVE topology. Moreover, the experimental results will be used for the validation of a reduced numerical model obtained via a nonlinear homogenization technique which allows for a significant reduction of the computational effort because the lattice specimen is modelled as an equivalent homogeneous anisotropic material. The objective is to develop a design approach to correctly reproduce the mechanical response of the LM, within larger structures, with reduced computational costs.

This study has been carried out in the framework of the $\mathrm{PhD}$ thesis "A dynamic behaviour study of architectured materials made by additive manufacturing", co-funded by the Commissariat à l'Energie Atomique et aux Energies Alternatives (CEA) and the Direction Générale de l'Armement (DGA). The samples were manufactured by ERPRO Group. The experiments were carried out at the materials durability experimental platform at the laboratory I2M of Bordeaux.

\section{References}

1. M. Montemurro, G. Bertolino, T. Roiné, A general multi-scale topology optimisation method for lightweight lattice structures obtained through additive manufacturing technology. Composite Structures. (2021). 258:113360.

2. H. Kazemi, A. Vaziri, J.A. Norato, Multi-material topology optimization of lattice structures using geometry projection. Computer Methods in Applied Mechanics and Engineering. (2020). 363:112895.

3. C. Qi, L-Z. Pei, A. Remennikov, S. Yang, J. Liu, J-S. Wang, X-W. Liao, Parametric study and optimization of the protect system containing a re-entrant hexagon cored sandwich panel under blast impact. Composite Structures. (2020). 252:112711.

4. G. Bertolino, M. Montemurro, G. De Pasquale, Multi-scale shape optimisation of lattice structures : an evolutionary-based approach. International Journal for Interactive Design and Manufacturing. (2019). 13:1565-1578.

5. L.J. Gibson, M.F. Ashby, Cellular Solids: Structure and properties - Second Edition, Cambridge Solid State Series. (1999).

6. A. Riot, E. Panettieri, M. Montemurro, S. Guerard, A. Coscuellela. Determination of energy absorption capacity of polymer lattice material from additive manufacturing. Mechanics of Advanced Materials and Structures. (2021) (submitted).

7. M. Jamshidian, N. Boddeti, D.W. Rosen, O. Weeger, Multiscale modelling of soft lattice metamaterials: Micromechanical nonlinear buckling analysis, experimental verification, and macroscale constitutive behaviour. International Journal of Mechanical Sciences. (2020). 188:105956. 